\title{
Vers une relance de l'anacarde au Mozambique
}

\author{
Jean-Paul LYANNAZ*
}

Cirad-Flhor, UPR Production fruitière, BP 1146, Garoua, Cameroun

jean-paul.lyannaz@cirad.fr

${ }^{*}$ Correspondance et tirés à part

Fruits, 2006, vol. 61, p. 125-133 (c) 2006 Cirad/EDP Sciences All rights reserved DOI: $10.1051 /$ fruits:2006011 www.edpsciences.org

RESUMEN ESPAÑOL, p. 133

\section{Towards a revival of cashew crops in Mozambique.}

Abstract - History. The cashew tree (Anacardium occidentale), originating in the Northeast of Brazil, was introduced into Mozambique at the end of the Xvith century. This country was for a long time the main world producer with 216000 t of nuts marketed in 1971-1972. Thereafter, certain political measures and the competition of the production from other countries made it lose its place. Facing this situation, actions to support the cashew nut crops have been undertaken since the Eighties. Revival of cashew crops in Mozambique. In Mozambique, the cashew tree is found all along the coast, but it is especially localized in the provinces of the North. The context of the liberalization and the privatization of the cashew operations carried out in recent years is described, as well as the consequences generated by these actions. The various projects to support the cashew operations especially relate to the production and the diffusion of seedlings. Most recently, the "Project of revival of cashew crops" (PRC) was elaborated in 1999. It aimed at the improvement of the cashew nut productivity and the production of quality nuts through operating systems ready to preserve the fertility of the soils. In this context, accompanying researches were focused on the disease and pest control of cashew nuts and on the selection of the best vegetable material. Disease control of cashew trees. The results obtained for oidium control with organic fungicides and for the possibility of an alternation of products (sulphur/triazoles) are presented. In the same way, the use of organic fungicides for anthracnose control is approached. Pest control. This point requires the training of an entomologist and the installation of a survey system which should in the long term make it possible to have a multilocal tool for an alarm and decision-making aid. Breeding and improvement. Obtaining new varieties was approached by a behavior clonal test, the selection of common local types and by hybridizations. Conclusions. The first PRC results are encouraging and its realization allowed the constitution of a regional research unit which is able to consolidate and instigate research on cashew nut crops on a national scale.

Mozambique / Anacardium occidentale / development projects / research projects / agricultural development / economic development / disease control / pests / plant breeding

\section{Vers une relance de l'anacarde au Mozambique.}

Résumé - Historique. L'anacardier (Anacardium occidentale), originaire du nord-est du Brésil, a été introduit au Mozambique à la fin du $\mathrm{XVI}^{\mathrm{e}}$ siècle. Ce pays a longtemps été le premier producteur mondial avec 216000 t de noix commercialisées en 1971-1972. Par la suite, certaines mesures politiques et la concurrence d'autres pays producteurs lui ont fait perdre sa place. Face à cette situation, des actions d'appui à la filière anacarde ont été entreprises à partir des années quatre-vingt. Relance de l'anacarde au Mozambique. Au Mozambique, l'anacardier se rencontre tout le long de la côte, mais il est surtout localisé dans les provinces du Nord. Le contexte de la libéralisation et de la privatisation de la filière engagées ces dernières années est décrit, ainsi que les conséquences engendrées par ces actions. Les différents projets d'appui à la filière ont surtout concerné la production et la diffusion de plants. Le plus récent, le "Projet de relance du cajou " (PRC) a été élaboré en 1999. Il a visé l'amélioration de la productivité de l'anacarde et la production de noix de qualité au travers de systèmes d'exploitation aptes à conserver la fertilité des sols. Dans ce contexte, les recherches d'accompagnement se sont focalisées sur la lutte contre les maladies et parasites de l'anacarde et la sélection de matériel végétal performant. Lutte contre les maladies de l'anacardier. Les résultats obtenus pour le contrôle de l'oïdium avec des fongicides organiques et la possibilité d'une alternance de produits (soufre / triazole) sont présentés. De même, l'utilisation de fongicides organiques pour le contrôle de l'anthracnose est abordée. Contrôle des ravageurs. Ce point nécessite la formation en cours d'un entomologiste et la mise en place d'un système d'avertissement qui devrait permettre à terme de disposer d'un outil multirégional d'alerte et d'aide à la décision. Sélection et amélioration. L'obtention de nouvelles variétés a été abordée par un essai de comportement clonal, la sélection de types communs locaux et la création d'hybrides. Conclusions. Les premiers résultats du PRC sont encourageants et sa réalisation a permis la constitution d'une unité de recherche régionale qui est venue conforter et redynamiser la recherche anacarde à l'échelle nationale.

Mozambique / Anacardium occidentale / projet de développement / projet de recherche / développement agricole / développement économique / contrôle de maladies / organisme nuisible / amélioration des plantes 


\section{Historique}

L'anacardier (Anacardium occidentale), encore appelé cajou, appartient à la grande famille des anacardiacées dont il existe environ 60 genres et 400 espèces, des essences tropicales, pour la plupart. Parmi les espèces les mieux connues, citons le manguier (Mangifera indica) et le prunier (Spondias monbin). C'est un arbre d'environ $10 \mathrm{~m}$ de hauteur à feuillage persistant. L'inflorescence, en panicule terminale, porte de nombreuses fleurs mâles ou hermaphrodites, fortement parfumées. Le fruit, (la noix) est un akène de (2 à 3 ) cm de longueur porté au bout d'un pédoncule renflé, la pomme cajou, qui est comestible, de goût sucré et légèrement astringent.

Cet arbre est originaire du nord-est du Brésil où il fut découvert par les explorateurs espagnols dans l'état de Maranha. Sa description et ses utilisations ont été énoncées pour la première fois par le naturaliste français Thevet [1] et les Portugais Gandão [2] et Soares de Soucer [3].

À la fin du XVI ${ }^{\mathrm{e}}$ siècle, les missionnaires portugais introduisent l'arbre en Angola, sur la côte Est africaine, dont au Mozambique, et dans leurs possessions en Inde. La production d'anacarde est donc ancienne au Mozambique et reste importante pour l'économie nationale.

Le Mozambique a longtemps été le premier producteur mondial avec une production record de 216000 t de noix commercialisées en 1971-1972. Comme la plupart des autres secteurs de l'économie, la filière s'est effondrée après l'indépendance et par suite de la guerre civile (18 000 t en 1983, 22000 t en 1989-1990). Le déclin a été provoqué par plusieurs facteurs qui ont conjugué leurs effets : mise en place de programmes de regroupement des populations en villages de l'époque socialiste, qui ont éloigné les propriétaires de leurs arbres; nationalisation de la filière qui s'est traduit par une rémunération non incitative du producteur; guerre civile, vieillissement progressif du verger, développement de maladies et dégâts de cyclones, le cyclone Nadia, en particulier, a affecté une partie non négligeable du verger de la province de Nampula en 1994.
La montée concomitante de la production d'autres pays producteurs ont fait perdre sa place au Mozambique : la production est passée de $43 \%$ de la production mondiale à la fin des années 70 à moins de $5 \%$ actuellement. Face à cette situation, des actions d'appui à la filière ont démarré à partir du milieu des années quatre-vingt, la dernière en date étant le "Projet de relance du cajou " (PRC / 2001-2006) financé par l'Agence française de développement (AFD).

\section{Relance de l'anacarde au Mozambique}

\subsection{Localisation de la production}

Au Mozambique, l'anacarde se rencontre tout le long de la côte du canal du Mozambique sur près de 2000 km et jusqu'à 200 km à l'intérieur des terres (figure 1).

Le principal bassin de production se situe dans les provinces du Nord (surtout la province de Nampula, mais également celles de Cabo Delgado et Zambézia) qui présentent des conditions agroécologiques favorables. L'anacardier y est un élément central, très répandu, du paysage agraire. Les provinces du nord sont aussi celles dont le verger est le plus âgé et le plus affecté par les maladies et dont les exploitations sont les plus petites, la province de Nampula étant plus densément peuplée.

La production est surtout le fait des petites exploitations familiales. Les producteurs du secteur familial se consacrent à la culture de l'anacarde et détiennent $90 \%$ du verger, estimé à $26 \mathrm{M}$ d'arbres [4]. Une partie importante de la production est autoconsommée, mais l'anacarde est aussi la culture de rente la plus importante du secteur familial. À l'échelle nationale, $40 \%$ des ménages ruraux possèdent des anacardiers, ce taux atteignant $80 \%$ dans les principales provinces productrices (Nampula, Gaza, Inhambane) où la quasi totalité des ménages récoltent des noix [5]. Les agriculteurs possèdent en général moins de 100 arbres (68 arbres par exploitation en moyenne sur la province de Nampula). La productivité moyenne est très faible, de l'ordre de (1 à 4) kg par arbre 
et par an, et hétérogène. Pour ces petites exploitations, l'anacarde est une source importante de revenus, même si les montants en jeu sont dans l'absolu très faibles : quelques dizaines de dollars par an.

Le champignon Ö̈dium anacardii (Powdery Mildiew Disease) et l'Helopeltis, insecte Miridae piqueur-suçeur, sont aujourd'hui les facteurs limitants les plus apparents de la production de cajou au Mozambique. Depuis quelques années cependant, l'anthracnose, Colletotrichum gloeosporioides, est une maladie qui tend à prendre un développement inquiétant.

\subsection{La libéralisation/privatisation de la filière}

Dans le cadre des programmes d'ajustement structurel engagés ces dernières années, la filière a été libéralisée et privatisée. À partir de 1991-1992 :

- les prix de commercialisation des noix de cajou ont été libérés et une véritable concurrence s'est installée pour l'achat des noix, sauf dans les zones très excentrées ;

- le parc d'usines de décorticage a été privatisé en un laps de temps limité et le taux de protection de l'industrie de décorticage nationale a été réduit. De ce fait, pour les exportations de noix brutes, il n'y a plus de restrictions quantitatives et les taxes sont passées de $26 \%$ en 1994-1995 à $14 \%$ en 1996-1997 avec une légère remontée à 18 \% en 1998.

Cette libéralisation/privatisation a engendré des turbulences, comme on l'observe classiquement dans les processus de libéralisation de filières de matières premières, mais elle a suscité une nette reprise des volumes commercialisés qui ont atteint 66500 t en 1995-1996 et, après une phase de stabilisation, 104300 t en 2004-2005.

À partir de 1999-2000, le développement de petites unités artisanales de transformation, qui se sont substituées aux grandes usines de décorticage, a permis d'augmenter la demande en noix brutes : 13870 t de noix ont été traitées dans ces unités en 2004 2005, la capacité totale de ces unités se situant actuellement autour de $21600 \mathrm{t}$ pour

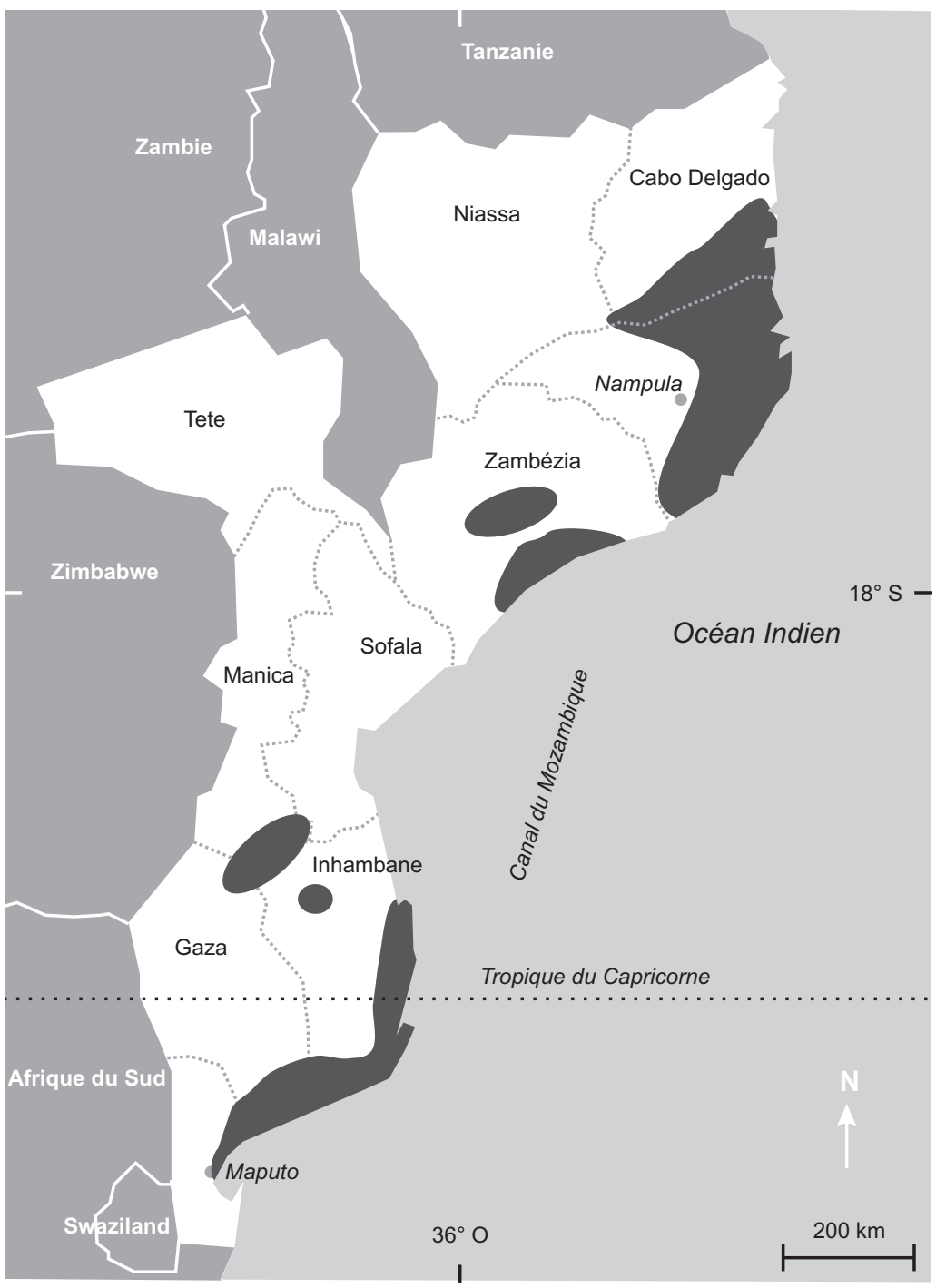

un total de 19 unités, dont 12 dans la seule province de Nampula.

\subsection{Les projets d'appui à la production}

Figure 1.

Zones de peuplements d'anacardiers au Mozambique [sources : Direcção Nacional de Geografia e Cadastro (DINAGECA), Mozambique].
Les récentes actions d'appui à la filière anacarde au Mozambique ont surtout concentré leur activité sur la production et la diffusion de plants, thème d'intervention le plus évident :

- "Projet de réhabilitation du cajou " : projet public financé par la Banque africaine de développement (BAD), qui a démarré au milieu des années quatre-vingt avec l'amorce 
d'un travail d'amélioration variétal au début des années quatre-vingt-dix.

- Programme de développement rural intégré mis en ouvre par l'ONG World Vision, qui a été interrompu à la fin 2001 pour le volet cajou; les trois sociétés agro-industrielles qui ont bénéficié d'un financement USAID lié au cyclone Nadia (Entreposto, Lomaco, JFS) entre 1995 et 1997 ont cessé leur activité de production de plants depuis l'achèvement de ce projet. Cependant, Entreposto a engagé un programme de sélection adaptative d'anacardiers nains issus de graines de clones améliorés importés du Brésil.

- L'ONG Aid Development from People to People (ADPP) qui gère un centre de formation à l'industrie du cajou et dispose d'une plantation près de Monapo.

Plus récemment, un " Projet de relance du cajou " (PRC) a été élaboré en 1999. Il s'est donné comme objectif la relance pérenne de la production mozambicaine de noix de cajou, à court et moyen terme, dans le cadre d'un dispositif équilibré de gestion concertée de la filière, associant l'État aux professionnels (producteurs, usiniers, commerçants). Ses actions ont été ciblées sur les districts de la province de Nampula, les plus importants en matière de production d'anacarde (districts d'Angoche, de Mogovolas et de Moma).

Ce projet, financé par l'Agence française de développement, s'inscrit dans le " plan directeur cajou " mis en place par la république du Mozambique en 1998. Il a pour maître d'ouvrage une structure du ministère de l'Agriculture et du développement rural, l'Instituto de Fomento do Caju (INCAJU). Il s'agit de stimuler la production de cajou, selon des démarches concertées et participatives, dans les exploitations où elle peut constituer un levier d'amélioration durable du revenu des producteurs.

Deux objectifs spécifiques principaux ont été définis :

- objectif 1 : améliorer les connaissances techniques et économiques sur les paramètres permettant d'accroître la productivité de l'anacarde et mener un programme de sélection de matériel végétal adapté ;

- objectif 2 : stimuler la production de cajou, en termes quantitatifs et qualitatifs, à court et à moyen terme, au travers de systèmes d'exploitation à la fois performants et équilibrés en termes d'insertion dans les systèmes d'exploitation de polyculture familiale, dans le respect de l'impératif de conservation de la fertilité.

Ces deux objectifs devront être atteints au travers de deux composantes :

- une composante de recherche appliquée confiée au Centre de coopération internationale en recherche agronomique pour le développement (Cirad) sous la tutelle du maître d'œuvre mozambicain : l'Instituto Nacional de Investigação Agronómica (INIA),

- une composante de vulgarisation et conseils aux exploitants, pour l'amélioration de l'exploitation de leurs vergers.

La poursuite de la recherche appliquée sur l'anacarde s'est révélée indispensable en raison d'un déficit des référentiels nécessaires pour étayer le conseil aux producteurs en matière de gestion du verger et, en particulier, de lutte contre les maladies et parasites de l'anacarde. Il existe également un besoin en matière de sélection de matériel végétal performant.

La synthèse des résultats obtenus entre 2001 et 2005 dans le cadre de cette composante de recherche appliquée fait l'objet du document présenté.

\section{Lutte contre les maladies de l'anacardier}

\subsection{Contrôle de l'oïdium}

L'ö̈dium de l'anacardier, dont l'agent responsable est évoqué dans la littérature sous le nom de Oïdium anacardii, est une maladie décrite dans plusieurs aires de production. Cette espèce est uniquement présente sous sa forme conidienne, aucun stade sexué n'ayant été observé jusqu'à présent.

Cette maladie apparaît durant la saison sèche. Les basses températures alliées à une hygrométrie importante en favorisent le développement. Les parties affectées, jeunes pousses, feuilles et surtout inflorescences, se couvrent d'un voile blanchâtre formé 
par le mycélium et les structures conidiennes du champignon. En période favorable à la maladie, l'attaque sur inflorescences peut provoquer une importante chute des fleurs capable de réduire la production à zéro dans certaines zones [6]. En Tanzanie, des études ont montré que l'oïdium pouvait être responsable de (50 à 70) \% des pertes de production [7]. Au Mozambique, c'est certainement la principale cause des faibles productions enregistrées ces dernières années.

\subsection{1. Étude de fongicides organiques}

L'usage de fongicides organiques pour le contrôle de l'oïdium de l'anacarde est assez récent. Les premiers essais ont comparé le soufre avec deux triazoles, le triadimenol et l'hexaconazole [8]. Les résultats positifs obtenus ont fait adopter ces deux substances actives pour un usage à grande échelle dans le pays.

Fort de ces premiers résultats, huit essais ont été effectués entre 2001 et 2004 dans le cadre du "Projet de relance du cajou ", soit en station, soit chez l'agriculteur. Il en a résulté :

- La démonstration de l'efficacité de molécules de familles différentes des classiques triazoles (strobilurine, spiroketalamine), ce qui permet de parer à toute éventualité de résistance induite par l'usage répété de triazoles.

- La démonstration de la double efficacité de la trifloxistrobine (strobilurine) contre l'oïdium et l'anthracnose. Une réduction des coûts de traitement serait donc possible.

- La confirmation de l'efficacité des génériques du Bayfidan (Shavit) et de l'Anvil (Volcano Richter) qui permettraient également une réduction substantielle des coûts.

Pour chacun de ces essais, le seuil d'intervention utilisé a été celui décrit par Topper et Madison [9] et les évaluations de la sévérité de la maladie ont été réalisées chaque semaine en utilisant une échelle de 0 à 6 [10]

\subsection{2. Étude d'une alternance de produits (soufre/triazole)}

Le soufre en poudre utilisé en traitement contre l'oïdium a montré son efficacité mais aussi ses limites du fait de ses effets sur l'aci- dification des sols, d'où l'idée de l'utiliser en alternance avec un triazole.

En raison des trop faibles niveaux d'infection de la maladie en 2004 dans la zone de l'essai, les résultats concernant une telle alternance des produits n'ont pas été significatifs et cet essai devrait donc être repris en 2005 .

\subsection{3. Étude épidémiologique de l'oïdium du cajou au nord du Mozambique}

La création d'une station météorologique sur la station de recherche agronomique de Nassuruma a permis de commencer en 2003 une étude de l'épidémiologie de l'oïdium du cajou dans la zone nord du Mozambique. Ce travail va se poursuivre dans les années à venir.

\subsection{Contrôle de l'anthracnose}

L'anthracnose est une maladie causée par Colletotrichum gloeosporioides. Sa première description au Mozambique date de 1958 [11]. La dissémination de la maladie intervient lorsque la température et l'humidité relative sont élevées, conditions réunies dans ce pays dès le début de la saison des pluies.

L'anthracnose est la deuxième cause de diminution des rendements après l'oïdium. Les dégâts occasionnés par cette maladie peuvent entraîner des pertes de rendement allant de (30 à 50) \% ainsi que des baisses de qualité des amandes [12].

Un premier travail, entrepris en collaboration avec le Naliendele Agricultural Research Institute (NARI, Tanzanie), a cherché à étudier une technique pour l'évaluation de la sévérité de l'anthracnose sur les feuilles.

\subsection{1. Étude de fongicides organiques}

Trois essais ont été réalisés entre 2003 et 2004 pour tester des fongicides organiques. Ils ont mis en évidence l'efficacité de trois molécules : la trifloxistrobine (Flint à $3 \mathrm{~g} \cdot \mathrm{L}^{-1}$ ), l'azoxistrobine (Ortiva à $6 \mathrm{~mL} \cdot \mathrm{L}^{-1}$ ) et le diphéniconazole (Score à $14 \mathrm{~mL} \cdot \mathrm{L}^{-1}$ ). La trifloxistrobine s'est révélée être une molécule à double efficacité contre l'oïdium et 
l'anthracnose, mais son coût rend encore son utilisation prohibitive.

\subsection{2. Étude préliminaire de l'épidémiologie de l'anthracnose du cajou au Mozambique}

Comme pour l'oïdium, la création d'une station météorologique sur la station de recherche agronomique de Nassuruma a permis de démarrer une étude de l'épidémiologie de l'anthracnose du cajou dans la zone nord du Mozambique. Ce travail va se poursuivre dans les années à venir dans le cadre de travaux de thèse.

\section{Contrôle des ravageurs}

Au-delà des problèmes liés aux maladies, certains insectes ravageurs peuvent constituer un problème grave s'ils ne sont pas contrôlés [13].

La formation en entomologie d'un jeune ingénieur sur la station de recherche du NARI (Tanzanie) a permis de commencer un programme d'étude d'une part sur la dynamique des populations des principaux parasites de l'anacarde au Mozambique (Helopeltis spp. et Pseudotherapus wayi) [13], d'autre part sur les possibilités d'adaptation d'un contrôle biologique à l'aide de la fourmi Oecophylla longinoda [14].

Cette formation devait être complétée, à partir de 2005, par la préparation d'un master en entomologie dans le cadre de l'Université Eduardo Mondlane de Maputo (capitale du Mozambique).

Enfin, un projet de système d'avertissement pour le contrôle des parasites est en cours d'élaboration avec le concours de techniciens chargés de la vulgarisation au sein du projet de relance du cajou lancé en 1999. Il devrait permettre à terme de disposer d'un outil multirégional d'alerte et d'aide à la décision.

\section{Sélections et amélioration}

La formation en amélioration variétale d'un ingénieur sur le centre de recherche de l'Empresa Brasileira de Pesquisa Agropecuária (Embrapa, centre de Fortaleza, Bré- sil) a permis, dès 2003 , de conforter le travail de sélection en cours et de débuter un programme d'amélioration.

Plusieurs axes d'études sont abordés : essai de comportement clonal, sélection de types communs locaux et amélioration par la création d'hybrides.

\subsection{Essais de comportement clonal}

Le suivi d'un essai clonal mis en place en 1998 a permis, après 6 années d'observations, de sélectionner quatre clones performants qui sont en cours d'enregistrement au niveau national. Par ailleurs, plusieurs essais multilocaux se poursuivent soit en milieu paysan (essais réduits au comportement de cinq clones dont quatre d'entre eux sont ceux sélectionnés précédemment, auxquels a été ajouté un clone local), soit en station.

L'essai en station, basé à Nassuruma au nord du Mozambique et installé en 2003, comporte 64 clones (types nains et communs) installés dans trois sites écologiques différents (provinces de Nampula, Zambezia et Inhambane). Outre les clones prometteurs de la station de Nassuruma, cet essai inclut les meilleurs clones, fruits de la recherche tanzanienne (20 clones de type commun), ainsi que cinq des meilleurs clones descendants de types nains brésiliens et sélectionnés par l'entreprise Entreposto (Monapo, Province de Nampula).

\subsection{Sélection de types communs locaux}

Un travail de sélection massale a été entrepris soit à partir d'une première sélection réalisée sur les différentes parcelles de la station de Nassuruma, soit à partir de sélections réalisées sur la base de l'expérience de propriétaires paysans dans différents districts de la province de Nampula.

\subsection{Amélioration par la création d'hybrides}

La technique adoptée pour effectuer les hybridations sur anacarde est celle couramment utilisée entre autre par la recherche brésilienne :

- identification des parents males et femelles, 
- choix, sur le parent femelle, d'une inflorescence bien développée avant l'ouverture des anthères, élimination de toutes les fleurs mâles et suppression de toutes les étamines des fleurs hermaphrodites,

- protection de l'inflorescence par ensachage,

- prélèvement de pollen sur les étamines des fleurs du parent mâle et dépôt sur les stigmates des fleurs femelles préalablement préparées en ayant soin de bien refermer le sachet autour de l'inflorescence après l'intervention,

- répétition de l'opération possible sur une même panicule car les stigmates ne sont pas tous réceptifs le même jour.

Les noix provenant des panicules ainsi traitées sont semées en pépinière pour une sélection préliminaire qui sera ensuite reprise en essai comparatif au champ.

Un premier travail portant sur de tels croisements a été entrepris en 2004 sur la station de Nassuruma. Les faibles résultats obtenus (six graines semées et germées sur 353 croisements réalisés) montrent la difficulté matérielle de réalisation de la technique; ils mettent en évidence la nécessité d'acquérir une expérience solide et d'assurer un suivi sans faille des inflorescences hybridées.

\section{Formation d'une équipe de chercheurs}

Après le recrutement de deux ingénieurs par l'INIA en 2003, le projet de relance du cajou au Mozambique s'est attaché à compléter la formation de ses chercheurs soit par des formations de courte durée (stage d'entomologie d'un mois sur le centre du Nari en Tanzanie, stage d'hybrideur et de sélectionneur de deux mois dans un centre Embrapa du Brésil), soit par des missions de contacts et d'échanges (Embrapa au Brésil, Nari en Tanzanie, Cirad en France), soit, enfin, par des formations diplômantes commencées en 2005 :

- maîtrise en entomologie réalisée dans le cadre de l'université Eduardo Mondlane à Maputo,

- Ph.D. en phytopathologie réalisé à l'Université de Prétoria (Afrique du Sud).
Durant son développement, le projet a par ailleurs bénéficié de l'appui de trois expertises techniques en amélioration, phytopathologie et entomologie de l'anacarde.

Actuellement, l'équipe mozambicaine de chercheurs nationaux est composée d'un phytopathologiste, d'un entomologiste, d'un sélectionneur dont la formation devra être complétée, et d'un agronome intervenant à l'interface de la recherche et du développement : formation des agents du développement aux différentes techniques de culture et de pépinière, suivi des essais et démonstration " on farm".

À noter que, dans le cadre du projet réalisé, les équipes de pathologie et d'entomologie ont bénéficié chacune de l'aménagement et de l'équipement d'un laboratoire.

\section{Conclusions}

Un premier travail a été réalisé dans le cadre du " projet de relance du cajou " au Mozambique ; il a donné des premiers résultats encourageants et a surtout permis la constitution d'une unité de recherche régionale qui est venue conforter et redynamiser la recherche anacarde à l'échelle nationale.

La campagne de production de noix de 2004-2005 ayant été particulièrement bonne (104 300 t), il peut être espéré un regain d'intérêt des populations paysannes pour cette culture et, de ce fait, une meilleure appropriation des résultats de la recherche et des innovations techniques proposées. Pour cela, une démarche d'analyse économique approfondie devra être menée pour démontrer clairement la viabilité des innovations face aux fluctuations du contexte économique mondial de l'industrie de l'anacarde.

Parallèlement, le développement de petites unités de transformation devrait contribuer à sécuriser la rentabilité de la culture en garantissant le prix et les quantités achetés.

Il peut être escompté que des financements " relais " interviendront après la fin de la prolongation du projet prévue pour fin 2006, afin de pérenniser ces recherches et les hisser au niveau international. Les priorités qui devraient être alors affichées 
peuvent se décliner en cohérence avec les différents thèmes abordés précédemment :

- analyses économiques pour valider l'intérêt des innovations techniques, en particulier celles relatives aux traitements phytosanitaires,

- caractérisation du/des Colletotrichum (ou autres) responsables des symptômes d'anthracnose observés au Mozambique (travail de thèse),

- poursuite du travail de fond sur l'étude de l'épidémiologie de l'anthracnose ; mise en évidence de seuils d'intervention (travail de thèse),

- poursuite du programme d'études sur les parasites de l'anacarde et développement de la lutte biologique,

- poursuite du programme d'amélioration et de sélection avec appui de missions de spécialistes pour épauler et parfaire la formation du chercheur impliqué,

- poursuite du positionnement de l'agronome en interface entre la recherche et la vulgarisation.

\section{Références}

[1] Thevet A., Les singularités de la France antarctique, autrement nommée Amérique, Les héritiers de Maurice de la Porte, Paris, France, 1557.

[2] Pero de Magalhães Gândavo, Tratado da terra do Brasil ; História da província Santa Cruz, Oficina de Antonio Gnsalvez, Lisboa, Portugal, 1576.

[3] Soares de Sousa G., Tratado descritivo do Brasil em 1587, Companhia Editora Nacional, Brasiliana 117, São Paulo, Brasil, 1971.

[4] Anon., Algunas reflexões sobre as intervenções num projecto em Nampula, Incaju, Maputo, Mozambique, 1999.

[5] Anon., Cashew production and marketing among smallholders in Mozambique: a gender-differentiated analysis based on household survey data, Preliminary report, Minist. l'Agric. Mozamb., Banq. Mond., Maputo, Mozambique, 1998.
[6] Mourichon X., Mission au Mozambique: maladie des anacardiers, proposition pour une lutte raisonnée, Irfa, rapp., Montpellier, France, 1991.

[7] Sijaona M.E.R., Shomari S.H., The powdery mildew disease of cashew in Tanzania, Dar es Salaam, Tanzanie, TARO Newsl. 11 (3) (1987) 4-5.

[8] Topper C.P., Boma F., Mhando H., Evaluation of fungicides for the control of powdery mildew (Oidium anacardii Noack) on cashew in Tanzania. A fungicide strategy development trials, in: Proc. Int. Cashew Coconut Conf., BioHybrids Internacional Ltd., Reading, UK, 1998, 254-259.

[9] Topper C.P., Maddison A.C., Manual de métodos de investigação de protecção de culturas para ser utilizado no caju. USAID/ Estratégias de melhoramento da producção e comercialização agrícola (AMIS II). Estudo de reabilitação do subsector do caju em Moçambique, USAID, Maputo, Mozambique, 1998.

[10] Nathaniel N.Q.R., Methods, including visual keys for assessment of cashew powdery mildew (Oidium anacardii Noack) severity, short commun., Int. J. Pest Manage. 42 (1996) 199205.

[11] Carvalho T., Mendes O., Doenças de plantas em Moçambique, Repartição de Sanidade Vegetal, Minerva Central, Lourenço Marques, Mozambique, 1958.

[12] Freire F.C.O., Cardoso J.E., Doenças do cajueiro, in: De Araujo J.P.P., da Silva V.V. (Eds.), Cajucultura. Modernas tecnicas de producção, EMBRAPA-CNPAT, Fortaleza, Brésil, 1995, 250-267.

[13] Boma F., Topper C.P., Stathers T., Population dynamics of Helopeltis spp. on cashew in southern Tanzania, in: Proc. Int. Cashew Coconut Conf., BioHybrids Internacional Ltd., Reading, UK, 1998, 185-187.

[14] Peng R., Christian K., Gibb K., Bioécologie of Helopeltis percinialis (Heteroptera: Miridae) with particular reference to the efficiency by Oecophyla smaragdina (Hymenoptera: Formicidae) in International Cashew and Coconut Conference, in: Proc. Int. Cashew Coconut Conf., BioHybrids Internacional Ltd., Reading, UK, 1998, 170-174. 


\section{Hacia un relanzamiento del anacardo en Mozambique.}

Resumen - Historia. El anacardo (Anacardium occidentale), originario del nordeste de Brasil, fue introducido en Mozambique a finales del siglo Xvi. Este país fue durante mucho tiempo el primer productor mundial con 216000 t de nueces comercializadas en 1971-1972. A continuación, ciertas medidas políticas y la competencia de otros países productores le hicieron perder su posición. Frente a esta situación, se emprendieron a finales de los años 80 sendas acciones de apoyo para la rama del anacardo. Relanzamiento del anacardo en Mozambique. En Mozambique, el anacardo se encuentra todo lo largo de la costa, pero se localiza sobre todo en las provincias del norte. Se describe el contexto de la liberalización y de la privatización de esta rama comprometida en estos últimos años, al igual que ocurre con las consecuencias derivadas de estas acciones. Los varios proyectos de apoyo para la rama se refirieron sobre todo a la producción y a la difusión de plantas. El más reciente, el "Proyecto de relanzamiento de la nuez de acajú" - "Projet de relance du cajou " (PRC) - se elaboró en 1999. Tuvo como objetivo la mejora de la productividad del anacardo y la producción de nueces de calidad a través de sistemas de explotación capaces de conservar la fertilidad de los suelos. En este contexto, las investigaciones de acompañamiento se focalizaron en la lucha contra las enfermedades y parásitos del anacardo y la selección de material vegetal con buenos resultados. Lucha contra las enfermedades del anacardo. Se presentan los resultados obtenidos para el control del oídio con los fungicidas orgánicos y la posibilidad de una alternancia de productos (azufre / triazol). Asimismo, se aborda el uso de fungicidas orgánicos para el control de la antracnosis. Control de las plagas. Este punto requiere la formación en curso de un entomólogo así como la instalación de un sistema de atención el cual, una vez llegado el momento, debería permitir disponer de una herramienta multiregional de alerta y de ayuda a la decisión. Selección y mejora. Se abordó la obtención de nuevas variedades mediante un intento de comportamiento clonal, la selección de tipos comunes locales y la creación de híbridos. Conclusiones. Los primeros resultados del proyecto PRC son esperanzadores y su realización permitió la constitución de una unidad de investigación regional que vino a confortar y a volver a dinamizar la investigación del anacardo a escala nacional.

Mozambique / Anacardium occidentale / proyectos de desarrollo / proyectos de investigación / desarrollo agrícola / desarrollo económico / control de enfermedades / plagas / fitomejoramiento 